\title{
Nachschlagewerk für Begutachtungen
}

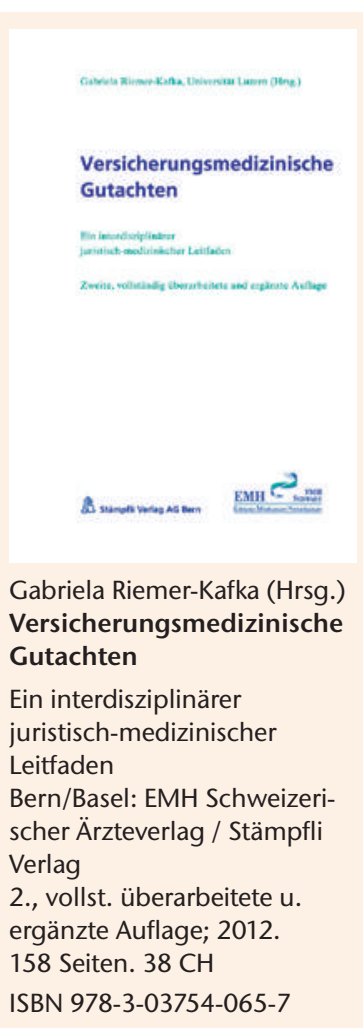

Das versicherungsmedizinische Gutachten ist ein Beweismittel, das in strittigen Fragen von Versicherungen, Gerichten oder den betroffenen Parteien beansprucht wird. Da die korrekte Entscheidungsfindung von der Plausibilität und Konsistenz des Gutachtens abhängt, sind inhaltliche und formale Anforderungen an ein solches zu definieren.

Der vorliegende juristisch-medizinische Leitfaden zeichnet sich dadurch aus, dass Ärzte und Juristen gemeinsam die Fragestellungen erarbeitet haben, die sich für die Erstellung von Gutachten ergeben. Er befasst sich mit Funktion und Modalitäten des Gutachtens, Aufgaben des Gutachters, sowie gutachtlich relevanten Begriffen.

Die Herausgeberin, Professorin für Sozialversicherungs- und Arbeitsrecht an der Universität Luzern und Initiantin eines interdisziplinären Arbeitskreises von Ärzten und Juristen, ist Autorin zahlreicher sozialversicherungsrechtlicher Publikationen und damit qualifiziert für die Herausgabe eines solchen interdisziplinären Werkes. Da erfahrungsgemäss Juristen und Mediziner grundsätzlich unterschiedlich denken und argumentieren, ist der Versuch einer Synthese dieser zwei Denkwelten als positiv einzustufen.

Das Ziel dieser interdisziplinären Gruppe bestand darin, offene Fragen und Verständnisschwierigkeiten zwischen Juristen und Medizinern zu analysieren und eine Abstimmung der gegenseitigen Erwartungen bezüglich medizinischer Gutachtenqualität zu schaffen. Das Resultat dieser Zusammenarbeit liegt nun in Gestalt dieses überarbeiteten Leitfadens vor.
Das erste Kapitel stellt die Funktion medizinischer Gutachten und die Aufgaben des ärztlichen Gutachters vor. Die Arzt-Patienten-Beziehung, die Methodik der Begutachtung und die Voraussetzungen, die an Gutachter und Gutachten gestellt werden, sind zunächst aus medizinischer Sicht dargestellt, und anschliessend werden die rechtlichen Beziehungen zwischen Patient, Entscheidungsträger und Gutachter beleuchtet, gefolgt von der Rechtsprechung des Bundesgerichts mit den konsekutiven Leitlinien zum Beweiswert und zur Beweiswürdigung medizinischer Gutachten.

Das zweite Kapitel bespricht die spezifischen Modalitäten eines versicherungsmedizinischen Gutachtens umfassend, dazu werden auch Fragen zu zeitlichen Aspekten der Begutachtung, zur optimalen Vorgehensweise bei fremdsprachigen Exploranden und zu Fragen des Datenschutzes behandelt.

Eine Auswahl wichtiger Begriffe wird im letzten Kapitel aus medizinischer und juristischen Perspektive zusammengefasst.

Insgesamt vermittelt der Leitfaden eine gute und nahezu vollständige Übersicht über die versicherungsmedizinische Begutachtung, fördert das Verständnis des juristischen Denkens für Mediziner und umgekehrt für Juristen und dient vorab als Nachschlagewerk für alle in der Begutachtung Engagierten (Mediziner, Richter, Anwälte, Versicherungsjuristen und Sachbearbeiter) für spezifische versicherungsmedizinische gutachtliche Fragen.

Hans Zollinger-Kies, Zürich 